\title{
The effect of asymmetric disorder on the diffusion in arbitrary networks
}

\author{
RÓBERT JUHÁSZ \\ Institute for Solid State Physics and Optics, Wigner Research Centre for Physics, H-1525 Budapest, P.O. Box 49, \\ Hungary
}

PACS 05.40.-a - Fluctuation phenomena, random processes, noise, and Brownian motion

PACS 05.70.Ln - Nonequilibrium and irreversible thermodynamics

PACS 64.60.aq - Networks

\begin{abstract}
Considering diffusion in the presence of asymmetric disorder, an exact relationship between the strength of weak disorder and the electric resistance of the corresponding resistor network is revealed, which is valid in arbitrary networks. This implies that the dynamics are stable against weak asymmetric disorder if the resistance exponent $\zeta$ of the network is negative. In the case of $\zeta>0$, numerical analyses of the mean first-passage time $\tau$ on various fractal lattices show that the logarithmic scaling of $\tau$ with the distance $l, \ln \tau \sim l^{\psi}$, is a general rule, characterized by a new dynamical exponent $\psi$ of the underlying lattice.
\end{abstract}

Introduction. - Disorder - an inevitable feature of nature - is known to induce striking slowing down phenomena in transport processes and in the relaxation of systems with many degrees of freedom [1-3]. The source ' of complexity in such systems is twofold. First, the dynamics can be regarded as a random walk in the configuration space, which is, in general, rather complicated. This problem also arises directly in the context of transport processes, which usually take place on inhomogeneous structures in reality 22. Second, the system is frequently subject to an external source of disorder, such as a random force-field, which can be modeled by quenched (i.e. time-independent) random transition rates. The theoretical description of the dynamics of such systems is a challenging task since, in most cases, the complexity induced by disorder makes the application of analytical techniques of clean systems extremely hard. Diffusion in the presence of the most general form of disorder, where transitions between pairs of states are non-symmetric, is non-trivial even on regular $d$-dimensional lattices. In the simplest case of $d=1$, where a single-valued potential exists, the position $x(t)$ of the walker varies ultra-slowly with time, obeying

$$
\overline{\left\langle x^{2}(t)\right\rangle} \sim(\ln t)^{2 / \psi},
$$

when the average force acting on the walker is zero 4 . Here, $\langle\cdot\rangle$ denotes an average over different stochastic histories, whereas the overbar denotes an average over the random transition rates. This is Sinai's diffusion law, where the value $\psi=1 / 2$ of the barrier exponent is related to the Gaussian fluctuations of the potential landscape. In higher dimensions, no potential can be defined, and the system is a genuinely non-equilibrium process. For increasing $d$, the effects of disorder are expected to be less pronounced as there are more and more paths connecting the initial and final state. Weak disorder expansions [5] and perturbative renormalization group analyses [6, 7] agree in that, for $d \geq d_{c}=2$, the diffusion remains normal, i.e. $\overline{\left\langle\mathbf{x}^{2}(t)\right\rangle} \sim t$, with logarithmic corrections at the critical dimension $d_{c}=2$, while for $d<2$ the disorder is relevant and results in sub-diffusion. Besides studying directly $x(t)$, the inverse question of how large the mean first-passage time (MFPT) from a given initial state to a final one is frequently asked in such systems [8]. In probing the dynamics, the finite-size scaling of the MFPT is an alternative possibility as, in general, it obeys the same dynamical relation between time and length scales as $x(t)$ does.

In transition networks other than regular lattices neither a general criterion for the relevance of asymmetric disorder nor the dynamics in case of relevance are known. Our aim in this letter is to study these questions in general. We will prove an exact relationship between the strength of weak asymmetry in the transition rates and the effective resistance of the corresponding resistor network, by which a relevance criterion can be formulated in terms of the sign of the resistance exponent $\zeta$. This will be then 
demonstrated by numerical calculations in various random and non-random fractal lattices. In the case of relevance $(\zeta>0)$, the dynamics are found to be logarithmic given by Eq. (11) with a barrier exponent $\psi$ that is characteristic of the underlying lattice.

The model and its renormalization. - To formulate the above statements precisely, let us assume that the system has a finite number of states labeled by the integers $i=1,2, \ldots, N$ and consider a continuous-time random walk on them, where the transition rates $\left(p_{i j}, p_{j i}\right)$ are from link to link independent random variables. The rates $p_{i j}$ and $p_{j i}$ are allowed to be different but their distributions are required to be identical, so that the average local 'force' is zero, i.e.

$$
\overline{F_{i j}} \equiv \overline{\ln \left(p_{i j} / p_{j i}\right)}=0
$$

on each link $(i j)$. For the sake of simplicity, we assume, furthermore, that every state is reachable from every other one. The central quantity in a fixed realization of the transition network is the MFPT, $\tau_{i}^{(A)}$, which is the expected value of the time needed to first reach any state in a fixed set $A$ of target sites when starting from state $i$. These quantities with different starting state $i$ obey the following backward master equation [8]:

$$
\sum_{i} p_{j i} \tau_{i}^{(A)}-\sum_{i} p_{j i} \tau_{j}^{(A)}=-K_{j}
$$

with $K_{i}=1$ for all $i$ and the boundary conditions $\tau_{i}^{(A)}=0$ for all $i \in A$. As it has been recently pointed out in ref. 9], this form of the equation enables one to calculate $\tau_{1}^{(A)}$ by recursively eliminating all states other than 1 and $A$ one after the other in a way closely related to strong disorder renormalization group (SDRG) methods [3]. When eliminating state $k$, direct transitions between states that were linked with state $k$ are generated with the rates:

$$
\tilde{p}_{i j}^{0}=p_{i k} p_{k j} / \sum_{i} p_{k i} . \quad \text { (generation) }
$$

If the link $(i j)$ has already existed before the elimination of $k$ than its rates $p_{i j}$ are added to the newly generated ones, so that the final renormalized rates read as:

$$
\tilde{p}_{i j}=p_{i j}+\tilde{p}_{i j}^{0} . \quad(\text { addition })
$$

The parameters $K_{i}$ at those states that were linked with state $k$ are also renormalized as $\tilde{K}_{i}=K_{i}+p_{i k} K_{k} / \sum_{i} p_{k i}$. When all states except of 1 and $A$ have been eliminated, the MFPT can be calculated by $\tau_{1}^{(A)}=\tilde{K}_{1} / \sum_{i \in A} \tilde{p}_{1 i} 1$. In the simple case $d=1$, the generation rule in Eq. (4) is the only operation and its product form results in a rapid decrease of effective rates under the elimination (or

\footnotetext{
${ }^{1}$ The denominator here has a direct probabilistic interpretation: $\sum_{i \in A} \tilde{p}_{1 i} / \sum_{i} p_{1 i}$ is the probability that the system, leaving state 1 , will reach state $A$ earlier than state 1 .
}

renormalization) procedure that signals slow dynamics. In more complex networks, also the addition rule given in Eq. (5) is applied since alternative paths from state $i$ to $j$ which do not go through $k$ may exist. The more connected the network is the more frequently the addition occurs and the slower the effective rate decreases under renormalization.

Renormalization of the weakly asymmetric model. - In general, the recursions described above cannot be solved analytically. Nevertheless, when merely seeking an answer to whether disorder is relevant, the analysis can be greatly simplified as follows. It is known in the case $d=1$ that any weak disorder drives the system to the infinite-randomness fixed point of the SDRG [3] that describes the logarithmic behavior in Eq. (11). Moreover, the behavior of the asymmetry in the effective transition rates alone reflects the logarithmic dynamics as $\left|\ln \left(\tilde{p}_{1 N} / \tilde{p}_{N 1}\right)\right| \sim N^{1 / 2}$, and indicates the relevance of weak disorder. Therefore, we shall deal only with a weak, asymmetric perturbation of the symmetric model of the form $p_{i j} / p_{j i} \equiv 1+\epsilon_{i j}$ with $\epsilon_{i j}$ infinitesimally small, and keep track of the renormalization of $p_{i j}$ and $\epsilon_{i j}$. The transformation rules then read in leading order in $\epsilon_{i j}$ as

$$
\begin{array}{rr}
\tilde{\epsilon}_{i j}^{0}=\epsilon_{i k}+\epsilon_{k j}, \quad & \text { (generation) } \\
\tilde{p}_{i j} \tilde{\epsilon}_{i j}=p_{i j} \epsilon_{i j}+\tilde{p}_{i j}^{0} \tilde{\epsilon}_{i j}^{0}, & \text { (addition) }
\end{array}
$$

whereas the rates $p_{i j}$ still obey Eqs. (415), however, they are now symmetric, $p_{i j}=p_{j i}$ and transform identically to the reduction rules of a resistor network with resistances $r_{i j} \equiv 1 / p_{i j}$ on links $(i j)$. Let us consider an ensemble of networks with fixed resistances $r_{i j}$ and random variables $\epsilon_{i j}$ on each link, for which Eq. (2) implies $\overline{\epsilon_{i j}}=0$, the overbar now denoting the average over $\epsilon_{i j}$ on link $(i j)$. In order to prove general statements we need to allow $\epsilon_{i j}$ to be non-identically distributed on different links and to require that $\overline{\epsilon_{i j}^{2}}=\alpha r_{i j}$, where $\alpha$ is an infinitesimally small global constant.

Relationship with the two-point resistance. First, let us consider a special class of networks which can be reduced to two fixed states, $a$ and $b$, by exclusively eliminating states with two links. An example is the hierarchical diamond lattice illustrated in Fig. 1 It is easy to see that, here, the generation and addition steps are equivalent to the well-known reduction of resistors in series and in parallel, respectively, and follow the simple rules:

$$
\begin{gathered}
\tilde{r}=r_{1}+r_{2}, \quad \tilde{\epsilon}=\epsilon_{1}+\epsilon_{2} \quad \text { (generation) } \\
\tilde{r}^{-1}=r_{1}^{-1}+r_{2}^{-1}, \quad \tilde{r}^{-1} \tilde{\epsilon}=r_{1}^{-1} \epsilon_{1}+r_{2}^{-1} \epsilon_{2} \quad \text { (addition). }
\end{gathered}
$$

When any of the above operations is performed, $\epsilon_{1}$ and $\epsilon_{2}$ are always independent, $\overline{\epsilon_{1} \epsilon_{2}}=0$, so one easily obtains that

$$
\overline{\tilde{\epsilon}_{i j}^{2}}=\alpha \tilde{r}_{i j}
$$

remains valid at any stage of the renormalization procedure, all the way to the last link connecting states $a$ and 
$b$ :

$$
\overline{\tilde{\epsilon}_{a b}^{2}}=\alpha \tilde{r}_{a b}
$$

For arbitrary networks, the elimination of states with more than two links cannot be avoided in general. When decimating a state with $n>2$ links, the relation Eq. (10) will be broken for the modified $n(n-1) / 2$ links, moreover, their rates will become correlated with each other. Surprisingly, when the network is reduced to two (arbitrary) states, $a$ and $b$, the relationship in Eq. (11) will still hold. In the following, it will be convenient to introduce the scaled asymmetry parameters $\omega_{i j} \equiv p_{i j} \epsilon_{i j}$, which satisfy initially $\overline{\omega_{i j}^{2}}=\alpha p_{i j}$. It is sufficient to prove Eq. (11) for complete networks (in which every state is linked with every other one) and for an arbitrary set of rates $\left\{p_{i j}\right\}$, then it applies to any other network by setting $p_{i j}=\overline{\omega_{i j}^{2}}=0$ for appropriate links, which amounts to deleting that link. For $N=3$, the statement is true since this network belongs to the special class, while for $N>3$ we shall prove it by induction. Assume the Eq. (11) holds for a fixed $N$. It follows from Eqs. (6) that the final asymmetry parameter is a linear combination of the initial ones: $\tilde{\omega}_{a b}^{(N)}\left(\left\{p_{i j}\right\}\right)=\sum_{i<j} C_{i j}^{(N)}\left(\left\{p_{i j}\right\}\right) \omega_{i j}$, where the coefficients are functions of the set of rates and the summation goes over all links. Using this, Eq. (11) can be rewritten as $\sum_{i<j}\left[C_{i j}^{(N)}\left(\left\{p_{i j}\right\}\right)\right]^{2} p_{i j}=\tilde{p}_{a b}^{(N)}\left(\left\{p_{i j}\right\}\right)$. Now let us extend the network to a one state larger one and keep the notation of parameters on links of the $N$-state subgraph, while denoting the rates and the scaled asymmetry parameters on the links from the old state $i$ to the new one by $q_{i}$ and $\phi_{i}, i=1,2, \ldots, N$, respectively. If the new state is eliminated, then effective rates $P_{i j}=q_{i} q_{j} / \sum_{i=1}^{N} q_{i}$ and scaled asymmetry parameters $\Omega_{i j}=\left(q_{j} \phi_{i}-q_{i} \phi_{j}\right) / \sum_{i=1}^{N} q_{i}$ are generated according to Eqs. (4) and (6) at all old links, and are added to the old parameters as given in Eqs. (5) and (17). This yields for the parameters of the system with $N+1$ states:

$$
\begin{aligned}
& \tilde{p}_{a b}^{(N+1)}\left(\left\{p_{i j}\right\},\left\{q_{i}\right\}\right)=\sum_{i<j} C_{i j}^{2}\left(p_{i j}+P_{i j}\right), \\
& \tilde{\omega}_{a b}^{(N+1)}\left(\left\{p_{i j}\right\},\left\{q_{i}\right\}\right)=\sum_{i<j} C_{i j}\left(\omega_{i j}+\Omega_{i j}\right),
\end{aligned}
$$

where we have used the shorthand notation $C_{i j}$ for $C_{i j}^{(N)}\left(\left\{p_{i j}+P_{i j}\right\}\right)$. It is to be shown that the expected value of the square of Eq. (13) is equal to Eq. (12) (multiplied by $\alpha$ ) for any $\left\{p_{i j}\right\},\left\{q_{i}\right\}$. Using that $\left\{\omega_{i j}\right\}$ and $\left\{\Omega_{i j}\right\}$ are independent and $\overline{\omega_{i j}^{2}}=\alpha p_{i j}$, we obtain that this is equivalent to

$$
\alpha \sum_{i<j} C_{i j}^{2} P_{i j}=\overline{\left(\sum_{i<j} C_{i j} \Omega_{i j}\right)^{2}} .
$$

Expanding $\Omega_{i j}$ in terms of $\phi_{i}$ and using $\overline{\phi_{i}^{2}}=\alpha q_{i}$, the r.h.s. assumes the form $\alpha \sum_{i=1}^{N} G_{i}^{2} q_{i}$ with the coefficients $G_{i}=\sum_{j \neq i} q_{j} C_{i j} / \sum_{j} q_{j}$, where $C_{i j}$ for $i>j$ is defined as $C_{i j} \equiv-C_{j i}$. Then Eq. (14) can be rewritten after some algebra in the form

$$
\sum_{i<j<k} q_{i} q_{j} q_{k}\left(C_{i j}+C_{j k}+C_{k i}\right)^{2}=0,
$$

where the summation goes over all triangles of links. A sufficient condition for Eq. (15) to hold is that

$$
C_{i j}^{(N)}\left(\left\{p_{i j}\right\}\right)+C_{j k}^{(N)}\left(\left\{p_{i j}\right\}\right)+C_{k i}^{(N)}\left(\left\{p_{i j}\right\}\right)=0
$$

for all triangles $i<j<k$ (and, in fact, for all closed paths) and for any set $\left\{p_{i j}\right\}$. Up to $N=4$ this can be justified by direct calculations. The key observation for the induction is that when extending the network by adding a new state then the coefficients of the old links in the enlarged network are modified only by a shift in their argument as $C_{i j}^{(N)}\left(\left\{p_{i j}\right\}\right) \rightarrow C_{i j}^{(N)}\left(\left\{p_{i j}+P_{i j}\right\}\right)$, see Eq. (13). Therefore the identity will hold in the enlarged network for all closed paths consisting of old links. The same network with $N+1$ states can, however, be built from another starting set of $N$ states by adding one extra state, as well, in which case the identity (16) can be extended to another set of links. It is easy to see that applying this reasoning to all possible sets of $N$ states (note that $a$ and $b$ are always part of this set), the old links cover all possible triangles of the extended network and the identity will be valid for $N+1$.

So, we have proved that Eq. (11) holds for arbitrary networks. This means that, although the two-point resistance depends on the particular network, the variance of the effective asymmetry parameter, which can be interpreted as the strength of disorder, depends on the resistance universally at least for weak disorder. If the resistance is increasing(decreasing) with the system size then the effective asymmetry is getting stronger(weaker) on larger scales. So, the dynamical relation between time and length scale on lattices with a decreasing resistance is expected to be stable against weak disorder; otherwise, it may be altered compared to that of the homogeneous system. Measuring the distance $l$ between $a$ and $b$ in terms of the linear size if the network can be embedded in a $d$-dimensional lattice and in terms of the length of shortest path otherwise, the resistance scales in many cases as $\tilde{r}_{a b}(l) \sim l^{\zeta}$, where $\zeta$ is the resistance exponent. The strength of disorder then scales with $l$ in leading order as

$$
\left|\ln \left(\tilde{p}_{a b} / \tilde{p}_{b a}\right)\right|=\left|\tilde{\epsilon}_{a b}\right| \sim \sqrt{\tilde{r}_{a b}} \sim l^{\zeta / 2} .
$$

For $\zeta>0$ this is the same type of logarithmic behavior as known for $d=1$, with the barrier exponent $\psi^{0}=\zeta / 2$. But unlike for $d=1$, this law will, in general, be modified for finite disorder by higher order correction terms which are expected to be non-universal. Indeed, numerical results that will be presented in the rest of this work indicate, in the case of $\zeta>0$, a logarithmic scaling law but with $\psi \neq \psi^{0}$.

The results obtained here are in accordance with previous ones on regular lattices. For $d>2$, the two-point resistance tends to a constant as $\tilde{r}_{a b}(l) \sim l^{\zeta}+$ const, with $\zeta=2-d<0$; at $d=2$, where the diffusion is normal with a logarithmic correction, it still increases but just barely (formally $\zeta=0$ ), while for $d=1, \zeta=1$. So, in general, we expect disorder to be relevant(irrelevant) if $\zeta>0(\zeta<0)$ while, in the marginal case $\zeta=0$, it will induce at most corrections to the clean behavior. 
Numerical analysis. - We have tested this criterion on fractal lattices, where, in the case of homogeneous rates, the law of diffusion is, in general, of the form $\left\langle\mathbf{x}^{2}(t)\right\rangle \sim t^{2 / d_{w}}$, characterized by an anomalous diffusion exponent, $d_{w}>2$ [1. This problem has been thoroughly investigated but, apart from early Monte Carlo simulation studies 10,11, not in the presence of asymmetric disorder. The Einstein relation between diffusion and conduction implies that $\zeta=d_{w}-d_{f}$, where $d_{f}$ is the fractal dimension. From this, one can see that $\zeta>0$ for all fractals in $d=2$ and, in fact, $\zeta$ is positive for most fractals.

We have numerically calculated the MFPT by recursively solving Eq. (3) on three different fractal lattices with $\zeta>0$, namely, the Sierpinski triangle (ST) shown in Fig. 1 and bond percolation clusters in 2 and 3 dimensions at the percolation threshold. In the former case, the MFPT from one of the tips of the largest triangle to the other two tips was considered, whereas in the latter case, percolating clusters that connect opposite $d-1$ dimensional surfaces of a $d$-dimensional cube have been generated (with periodic boundaries in the other directions), and the MFPT from a given site on one surface to the opposite surface was considered. The rates on each
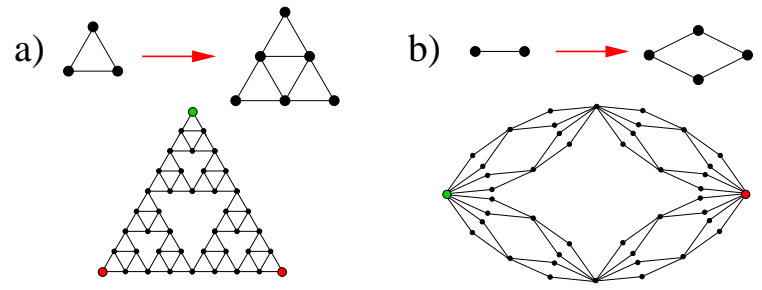

Fig. 1: Iteration rule and the 4th generation of the Sierpinski (a) and the hierarchical diamond lattice (b). The starting(target) sites are denoted by green(red) dots.

link were $p_{i j}=1, p_{j i}=\lambda<1$ with a random (equiprobable) orientation. The distributions of the logarithm of the MFPT determined in $10^{6}$ realizations for different linear system sizes $L$ are found to broaden with $L$ in all three cases, see Figs. 2,3 and to obey the scaling law

$$
\rho\left[\ln \left(\tau / \tau_{0}\right), L\right]=L^{-\psi} \tilde{\rho}\left[\ln \left(\tau / \tau_{0}\right) L^{-\psi}\right],
$$

where the barrier exponents $\psi$ are independent of the strength of disorder $\lambda$, and are estimated for the ST and $d=2$ and $d=3$ percolation clusters to be, in order, $0.296(2), 0.46(2)$ and $0.63(1)$.

We have the most accurate estimate for the ST, due to an efficient renormalization, essentially by reversing the construction procedure. Here, the barrier exponent $\psi=$ $0.296(2)$ can be seen to be significantly different from $\psi_{0}=$ $\zeta / 2=\ln (5 / 3) / \ln 4 \approx 0.368$.

Concerning marginal structures $(\zeta=0)$, we have considered the hierarchical diamond lattice (see Fig. 11), where $d_{f}=d_{w}=2$ and, in fact, $\tilde{r}_{a b}=1$ in each generation. The distribution of $\tau / L^{d_{w}}$ is found to converge to a limit distribution for $L \rightarrow \infty$, but very slowly; the

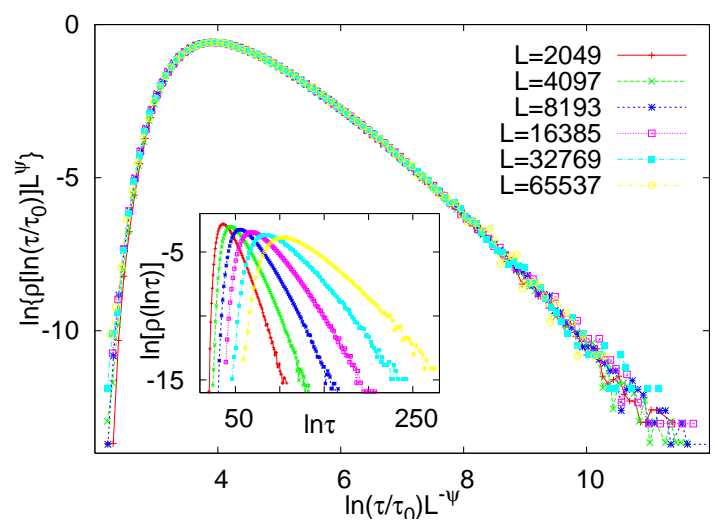

Fig. 2: Scaling plot of the distribution of the logarithm of the MFPT on Sierpinski triangles with different linear size $L$. The parameters are $\psi=0.296$ and $\ln \tau_{0}=-1.47$. The strength of disorder was $\lambda=0.1$. Inset: Unscaled distributions.

typical MFPT, $\tau_{\text {typ }} \equiv \exp (\overline{\ln \tau})$, has a logarithmic correction of the same form as in $d=2$ regular lattices: $L^{d_{w}} / \tau_{\text {typ }} \simeq D\left(1+a / \ln \tau_{\text {typ }}\right)$, see Fig. 目

Discussion. - In summary, we have revealed a close relationship between the effective strength of asymmetric disorder in the diffusion problem and the electric resistance. This yields a simple relevance criterion in terms of the resistance exponent. If $\zeta<0$, the dynamics are stable against weak asymmetric disorder, while if $\zeta>0$, they are unstable and, as numerical results show, the logarithmic scaling is not a peculiarity of one dimension but it is the general rule, characterized by the exponent $\psi$ of the underlying structure that is independent of other known dynamical exponents. In the marginal case, $\zeta=0$, slow corrections to the clean system behavior are expected to be general.

As aforementioned, stochastic processes can be regarded as random walks in their configuration spaces. In disordered stochastic systems with many degrees of freedom, logarithmically slow dynamical behavior indeed appears, such as in many-particle transport processes with zerorange or exclusion interaction [12, or in the contact process at criticality in one 13 and higher 14 dimensions. The effect of disorder is, however, not restricted to appear in one single (critical) point of the parameter space of these systems (including the random walk in one dimension) but the latter point is surrounded by an extended phase, called Griffiths phase [15, where the dynamical exponents are finite and vary with the parameters of the model non-universally [3]. In the random walk representation, the average force acting on the walker becomes non-zero in some direction in this phase, therefore this is out of the scope of the present treatment (cf. Eq. (2)). Nevertheless, it is plausible to expect systems with a positive resistance exponent to have a Griffiths phase, when a biased force-field is applied. The non-universal behavior with a finite dynamical exponent is, however, suppressed 


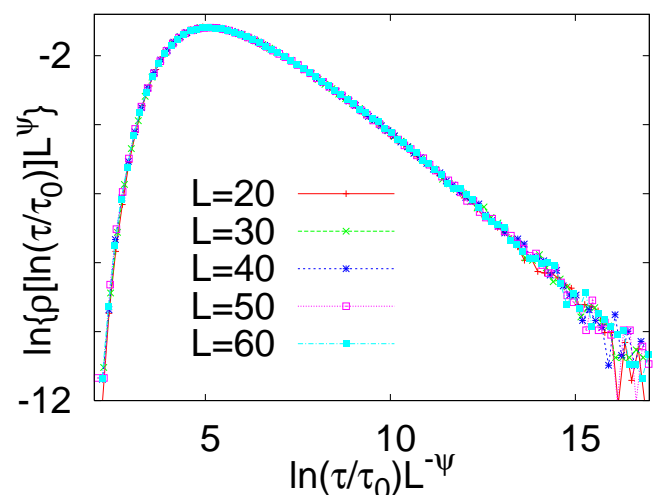

Fig. 3: The same plot for $d=3$ percolation clusters as in Fig. 2 with $\psi=0.63$ and $\ln \tau_{0}=-5.7$.

if the lattice contains macroscopic 'dead-ends' (such as in critical percolation clusters) from which the walker escapes after an exponentially large trapping time [1, leading to logarithmically slow dynamics also in the driven phase. The analyses of these phenomena is left for future research.

This work was supported by the János Bolyai Research Scholarship of the Hungarian Academy of Sciences and by the National Research Fund under grant no. K75324.

\section{REFERENCES}

[1] Havlin S. and Ben-Avraham D., Adv. Phys., 36 (1987) 695.

[2] Bouchaud J.P. and Georges A., Phys. Rep., 195 (1990) 217.

[3] Iglói F. and Monthus C., Phys. Rep., 412 (2005) 277.

[4] Sinai Ya.G., Theory Probab. Appl., 27 (1982) 256.

[5] Derrida B. and Luck J.M., Phys. Rev. B, 28 (1983) 7183.

[6] Luck J.M., Nucl. Phys. B, 225 (1983) 169.

[7] Fisher D.S., Phys. Rev. A, 30 (1984) 960.

[8] Redner S., A Guide to First-Passage Processes (Cambridge) 2001.

[9] Monthus C. and Garel T., J. Phys. A: Math. Theor., 43 (2010) 095001.

[10] Pandey R.B., J. Phys. A: Math. Gen., 20 (1987) L337.

[11] Majhofer A. and Cieplak M., J. Phys. A: Math. Gen., 21 (1988) 3481.

[12] Juhász R., Santen L. and Iglói F., Phys. Rev. Lett., 94 (2005) 010601.

[13] Hooyberghs J., Iglói F. and Vanderzande C., Phys. Rev. Lett., 90 (2003) 100601.

[14] Vojta T., Farquhar A. and Mast J., Phys. Rev. E, 79 (2009) 011111.

[15] Griffiths R.B., Phys. Rev. Lett., 23 (1969) 17.

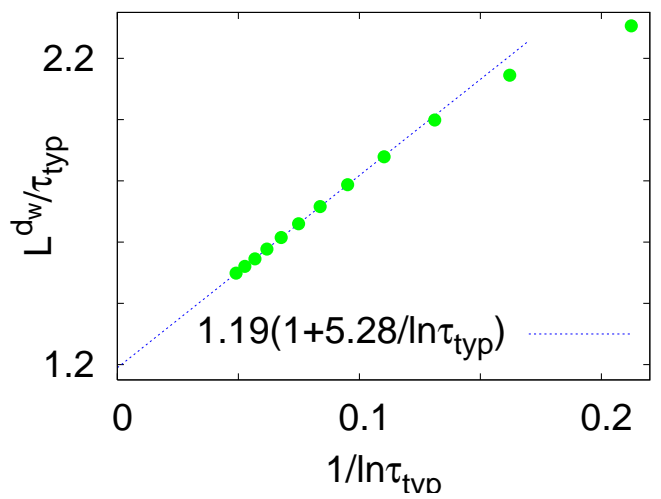

Fig. 4: Finite-size behavior of the typical MFPT in the hierarchical diamond lattice with $\lambda=0.1$. 